\title{
Anderson Localization of Electromagnetic Waves in Dielectric Media: Model Studies
}

\author{
M. Rusek And A. Ortowski \\ Institute of Physics, Polish Academy of Sciences \\ al. Lotników 32/46, 02-668 Warsaw, Poland
}

\begin{abstract}
Anderson localization of electromagnetic waves in random arrays of dielectric cylinders confined within a planar metallic waveguide is studied. The disordered dielectric medium is modeled by a system of randomly distributed $2 \mathrm{D}$ electric dipoles. An effective theoretical approach based on the method of images is developed. A clear distinction between isolated localized waves (which exist in finite media) and the band of localized waves (which appears only in the limit of the infinite medium) is presented. The Anderson transition emerging in the limit of an infinite medium is observed both in finite size scaling analysis of transmission and in the properties of the spectra of some random matrices. The sound physical interpretation of the obtained results suggests deeper insight into the existing experimental and theoretical work.
\end{abstract}

PACS numbers: 42.25.Fx, 42.25.Hz, 72.10.Fk, 78.20.Ci

\section{Introduction}

The concept of the Anderson localization originates from the studies of electron transport in disordered solids. This phenomenon is based completely on interference effects in multiple scattering of electron wave functions by a random potential. Several generalizations of the Anderson localization to other types of waves have been proposed. A convincing experimental demonstration that the Anderson localization of electromagnetic waves is possible in three-dimensional disordered dielectric structures has been given recently [1]. The strongly scattering medium has been provided by semiconductor powders with a very large refractive index. By decreasing the average particle size it was possible to observe a clear transition from linear scaling of transmission $\left(T \propto L^{-1}\right)$ to an exponential decay $\left(T \propto \mathrm{e}^{-L / \xi}\right)$. Some localization effects have been also reported in previous experiments on microwave localization in copper tubes filled with metallic 
and dielectric spheres [2]. However, the latter experiments were plagued by large absorption, which makes the interpretation of the data quite complicated.

Another experiment on microwave localization has been performed in a two-dimensional medium [3]. The scattering chamber was set up as a collection of dielectric cylinders randomly placed between two parallel aluminum plates on half the sites of a square lattice. These authors attributed the observed sharp peaks of transmission to the existence of localized modes and measured the energy density of the electromagnetic field localized by their random structures.

In this paper we present a simple model of the Anderson localization. It is applied to a problem of electromagnetic wave propagation in a disordered quasi one-dimensional medium. The random system under consideration consists of dielectric cylinders placed inside a metallic planar waveguide. The Anderson transition emerging in the limit of an infinite medium is observed both in finite size scaling analysis of transmission and in the properties of the spectra of some random matrices.

\section{Basic assumptions}

In the following we study the properties of the stationary solutions of the Maxwell equations in two-dimensional media consisting of randomly placed parallel dielectric cylinders of infinite height (i.e., very long as compared to the wavelength of the electromagnetic field). This means that one, say $(y)$, out of three dimensions is translationally invariant and only the remaining two $(x, z)$ are random. The main advantage of this two-dimensional approximation is that we can use the scalar theory of electromagnetic waves [4]:

$$
\boldsymbol{E}(\boldsymbol{r}, t)=\operatorname{Re}\left[\boldsymbol{e}_{y} \mathcal{E}(x, z) \mathrm{e}^{-\mathrm{i} \omega t}\right] .
$$

Consequently, the polarization of the medium takes the form

$$
\boldsymbol{P}(\boldsymbol{r}, t)=\operatorname{Re}\left[\boldsymbol{e}_{y} \mathcal{P}(x, z) \mathrm{e}^{-\mathrm{i} \omega t}\right] .
$$

Localization of electromagnetic waves in disordered 2D media is usually studied experimentally in microstructures consisting of dielectric cylinders with diameters and mutual distances being comparable to the wavelength [3]. However, it seems to be a reasonable assumption that what really counts for the basic features of localization is the scattering cross-section and not the real geometrical size of the scatterer itself. Therefore we will represent the dielectric cylinders located at the points $\left(x_{a}, z_{a}\right)$ by single $2 \mathrm{D}$ electric dipoles

$$
\mathcal{P}(x, z)=\sum_{a=1}^{N} p_{a} \delta^{(2)}\left(x-x_{a}, z-z_{a}\right) .
$$

It should be stressed that the boundary conditions considered in this paper are different from those encountered in the experiment of Ref. [3]. To minimize the effect of the waves reflected off the edges of the scattering chamber, its perimeter was lined with a layer of microwave absorber. Therefore, to model that particular 
experiment, it is appropriate to use the free space boundary conditions (as we did in our previous papers $[4,5])$.

\section{Planar waveguide}

In the present model we place the disordered dielectric medium between two infinite, perfectly conducting mirrors described by the equations $x=0$ and $x=d$. Thus we will consider the case where the cylinders are oriented parallel to the mirrors. For simplicity our discussion will be restricted to TE modes polarized along the $y$ axis only.

The electric field of the electromagnetic wave incident on the cylinders

$$
\mathcal{E}^{(0)}(x, z)=\sum_{m=1}^{M} \iota_{m} \mathcal{E}^{(m)}(x, z),
$$

may be expanded into the guided modes of the waveguide [6]:

$$
\mathcal{E}^{(m)}(x, z)=\frac{2}{\sqrt{\beta_{m} d}} \sin \left(\alpha_{m} x\right) \mathrm{e}^{\mathrm{i} \beta_{m} z}
$$

where the propagation constants are given by

$$
\alpha_{m}=\frac{\pi}{d} n, \quad \beta_{m}=\sqrt{k^{2}-\alpha_{m}^{2}} .
$$

The total field that can be measured far from the cylinders is fully described by the reflection $\rho_{m}$ and transmission $\tau_{m}$ coefficients of all guided modes

$$
\begin{aligned}
& \mathcal{E}(x, z)=\sum_{m=1}^{M} \iota_{m} \mathcal{E}^{(m)}(x, z)+\sum_{m=1}^{M} \rho_{m} \mathcal{E}^{(m) *}(x, z) \quad \text { for } \quad z \rightarrow-\infty \\
& \mathcal{E}(x, z)=\sum_{m=1}^{M} \tau_{m} \mathcal{E}^{(m)}(x, z) \text { for } z \rightarrow+\infty
\end{aligned}
$$

Using the Lorentz theorem and repeating the straightforward calculations (see, e.g., [6]) we easily arrive at the following expressions determining the transmission coefficients:

$$
\tau_{m}=\iota_{m}+\mathrm{i} \pi k^{2} \sum_{a=1}^{N} p_{a} \mathcal{E}^{(m) *}\left(x_{a}, z_{a}\right),
$$

and the reflection coefficients

$$
\rho_{m}=\mathrm{i} \pi k^{2} \sum_{a=1}^{N} p_{a} \mathcal{E}^{(m)}\left(x_{a}, z_{a}\right),
$$

for given dipole moments $p_{a}$. In the following sections we will relate $p_{a}$ to the values of the incident field calculated at the positions of the cylinders $\mathcal{E}^{(0)}\left(x_{a}, z_{a}\right)$.

\section{Method of images}

A simple way to take into account the boundary conditions of parallel mirrors and their influence on the electromagnetic field is to use the method of images. This technique has been used, i.e., in quantum electrodynamics (QED) calculations 
of spontaneous emission in cavities $[7,8]$. To reproduce the correct boundary conditions on the radiation field of each cylinder (3) the mirrors are replaced by an array of image cylinders whose phases alternate in sign

$$
\mathcal{P}(x, z)=\sum_{a=1}^{N} \sum_{j=-\infty}^{\infty}(-1)^{j} p_{a} \delta^{(2)}\left(x-x_{a}^{(j)}, z-z_{a}\right)
$$

where

$$
x_{a}^{(j)}=(-1)^{j} x_{a}+j d .
$$

Thus a finite system of dielectric cylinders (3) placed within a metallic waveguide is fully equivalent to an infinite system of cylinders (11) forming a slab in a free space. This fact allows us to utilize some results from our previous paper concerning dielectric cylinders in free space [4].

\section{Breit-Wigner scatterers}

The cylinders necessarily have an internal structure. For example to model a simple scattering process with one internal Breit-Wigner type resonance one can use the Breit-Wigner model of scattering. This gives the following form of the coupling between the dipole moment $p_{a}$ and the electric field incident on the cylinder $\mathcal{E}^{\prime}\left(x_{a}, z_{a}\right)$ :

$$
\mathrm{i} \pi k^{2} p_{a}=\frac{\gamma_{0}}{\left(\omega-\omega_{0}\right)+\mathrm{i} \gamma_{0}} \mathcal{E}^{\prime}\left(x_{a}, z_{a}\right) .
$$

The total scattering cross-section $\sigma$ [4] takes then the familiar Lorentzian form

$$
k \sigma=\frac{4 \gamma_{0}^{2}}{\left(\omega-\omega_{0}\right)^{2}+\gamma_{0}^{2}} .
$$

\section{Multiple scattering}

In the case of a confined medium the field acting on the $a$-th cylinder $\mathcal{E}^{\prime}\left(x_{a}, z_{a}\right)$ from Eq. (13) is the sum of the incident guided mode $\mathcal{E}^{(0)}$, which obeys the Maxwell equations in an empty waveguide, and waves scattered by all other cylinders and by all images

$$
\mathcal{E}^{\prime}\left(x_{a}, z_{a}\right)=\mathcal{E}^{(0)}\left(x_{a}, z_{a}\right)+\frac{\gamma_{0}}{\left(\omega-\omega_{0}\right)+\mathrm{i} \gamma_{0}} \sum_{b=1}^{N} G_{a b} \mathcal{E}^{\prime}\left(x_{b}, z_{b}\right), \quad a=1, \ldots N .
$$

Thus, in the present model the $G$ matrix from Eq. (15) needs to be defined differently than in Ref. [4]:

$$
\mathrm{i} \pi G_{a b}=2 \sum_{\rho_{a b}^{(j)} \neq 0}(-1)^{j} K_{0}\left(-\mathrm{i} k \rho_{a b}^{(j)}\right),
$$

where

$$
\rho_{a b}^{(j)}=\sqrt{\left(x_{a}-x_{b}^{(j)}\right)^{2}+\left(z_{a}-z_{b}\right)^{2}},
$$

denotes the distance between the $a$-th cylinder and the $j$-th image of the $b$-th 
cylinder and $K_{0}$ is the modified Bessel function of the second kind. Let us note that summation in Eq. (16) is performed over all $j$, for which $\rho_{a b}^{(j)} \neq 0$.

The system of linear Eqs. (15) fully determines the field acting on each cylinder $\mathcal{E}^{\prime}\left(x_{a}, z_{a}\right)$ for a given field of the guided mode $\mathcal{E}^{(0)}\left(x_{a}, z_{a}\right)$ incident on the system. Analogous relationships between the stationary outgoing wave and the stationary incoming wave are known in the general scattering theory as the Lippmann-Schwinger equations [9]. If we solve Eqs. (15) and use Eqs. (13) to find $p_{a}$, then we are able to find the transmission and reflection coefficients given by Eqs. (9) and (10).

\section{Localized waves}

By definition, an electromagnetic wave is localized in a certain region of space if its magnitude is (at least) exponentially decaying in any direction from this region. We will show now that electromagnetic waves localized in the system of dielectric cylinders placed in a planar metallic waveguide correspond to nonzero solutions $\mathcal{E}_{l}^{\prime}\left(x_{a}, z_{a}\right) \neq 0$ of Eqs. (15) for the incoming wave equal to zero, i.e., $\mathcal{E}^{(0)}(x, z) \equiv 0$. Let us note that we added an index $l$ which labels the localized waves.

Indeed, let us suppose that the field is exponentially localized. This means that there are no guided modes in the radiation field. Therefore (as shown in the previous section) Eq. (24) holds. Using Eq. (13) we see that the vector formed by the values of the field acting on the cylinders is orthogonal to the vector formed by the values of incident field calculated at the positions of the cylinders

$$
\sum_{a=1}^{N} \mathcal{E}_{l}^{\prime}\left(x_{a}, z_{a}\right) \mathcal{E}^{(0) *}\left(x_{a}, z_{a}\right)=0 .
$$

But simultaneously $\mathcal{E}_{l}^{\prime}\left(x_{a}, z_{a}\right)$ is a solution of a system of linear Eqs. (15) where $\mathcal{E}^{(0)}\left(x_{a}, z_{a}\right)$ is the right-hand side. Therefore $\mathcal{E}_{l}^{\prime}\left(x_{a}, z_{a}\right)$ is also a solution of Eqs. (15) with $\mathcal{E}^{(0)}\left(x_{a}, z_{a}\right) \equiv 0$.

The proof works also the other way round. Let us suppose that $\mathcal{E}_{l}^{\prime}\left(x_{a}, z_{a}\right)$ is a solution of Eqs. (15) for $\mathcal{E}^{(0)}\left(x_{a}, z_{a}\right) \equiv 0$. As the considered medium is non-dissipative, the time average energy stream integrated over a closed surface surrounding it must vanish. This means that there are again no guided modes in the radiation field (which in the case $\mathcal{E}^{(0)}(x, z) \equiv 0$ is equal to the total field). Therefore Eq. (24) holds and the wave is localized.

\section{Eigenproblem}

Let us note that for the incoming wave equal to zero, i.e., $\mathcal{E}^{(0)}(x, z) \equiv 0$, the system of Eqs. (15) is equivalent to the eigenproblem for the $G$ matrix

$$
\sum_{b=1}^{N} G_{a b} \mathcal{E}_{l}^{\prime}\left(x_{b}, z_{b}\right)=\lambda_{l} \mathcal{E}_{l}^{\prime}\left(x_{a}, z_{a}\right),
$$

where 


$$
\operatorname{Im} \lambda_{l}=\frac{\omega_{l}-\omega}{\gamma_{0}}
$$

Let us stress that Eq. (19) can be fulfilled only if the real part of an eigenvalue satisfies

$$
\operatorname{Re} \lambda_{l}=-1 .
$$

Therefore only those eigenvectors $\mathcal{E}_{l}^{\prime}\left(x_{a}, z_{a}\right)$ of the $G$ matrix which correspond to the eigenvalues $\lambda_{l}$ satisfying the condition (21) may be related to localized waves. Moreover, those waves can exist only for discrete frequencies $\omega_{l}$ given by Eq. (20). As discussed in the previous sections they are the same values of $\omega$ for which the transmission is equal to unity.

\section{Resonance poles}

Localized waves exist only if the geometry of the system (i.e., the positions of the cylinders and/or the thickness of the waveguide) meets certain specific requirements. However, it is always possible to look for resonances. A way of dealing with resonances in our formalism is to look for resonance poles in the complex frequency plane. Resonance poles are complex frequencies for which it is possible to solve Eqs. (15) as homogeneous equations, i.e., for the incoming wave $\mathcal{E}^{\prime}\left(x_{a}, z_{a}\right)$ equal to zero. The real and imaginary parts of the corresponding resonance frequencies determine then the positions $\omega$ and widths $\gamma$ of the resonances.

In the particular case of the Breit-Wigner type scatterers the real and imaginary parts of the eigenvalues of the $G$ matrix have a nice physical interpretation: they are equal to the relative widths $\left(\gamma-\gamma_{0}\right) / \gamma_{0}$ and positions $\left(\omega-\omega_{0}\right) / \gamma_{0}$ of the resonances. Indeed, using the explicit form of the complex frequency $\omega \rightarrow \omega-\mathrm{i} \gamma$ and comparing Eqs. (15) and (19) we get

$$
\omega-\mathrm{i} \gamma=\omega_{0}-\mathrm{i} \gamma_{0}[1+\lambda(\omega-\mathrm{i} \gamma)]
$$

This system of two coupled nonlinear equations determines the values of the resonance poles $\omega-\mathrm{i} \gamma$. In many physically interesting cases Eqs. (22) can be solved numerically by iteration.

Most localization experiments are performed in the range of optical or microwave frequencies. In this case usually $\gamma_{0} / \omega_{0} \ll 1$. For instance in solving it up to the first order in $\gamma_{0}$ one substitutes $\lambda\left(\omega_{0}\right)$ for $\lambda(\omega-\mathrm{i} \Gamma)$ getting

$$
\operatorname{Re} \lambda\left(\omega_{0}\right) \simeq \frac{\gamma-\gamma_{0}}{\gamma_{0}}, \quad \operatorname{Im} \lambda\left(\omega_{0}\right) \simeq \frac{\omega-\omega_{0}}{\gamma_{0}}
$$

\section{Single scattering}

Let us begin by considering a single dielectric cylinder placed in a metallic waveguide. The waveguide may introduce its own phase shift depending on the position of the cylinder with respect to its walls. This results in changing the width and position of the transmission and reflection resonance. 
Indeed, as an example let us consider a cylinder placed between the mirrors separated by a distance $k d=3 \pi / 2$. We have calculated numerically the corresponding $1 \times 1 G$ matrix (16). The images of the cylinder from Eq. (11) were summed from $j=-50000$ to $j=50000$. The resulting "spectrum" $\left(\lambda=G_{11}\right)$ of the $G$ matrix is plotted in Fig. 1b. We see that as opposed to the free-space case $\left(G_{11}=0\right)$ it is nonzero.
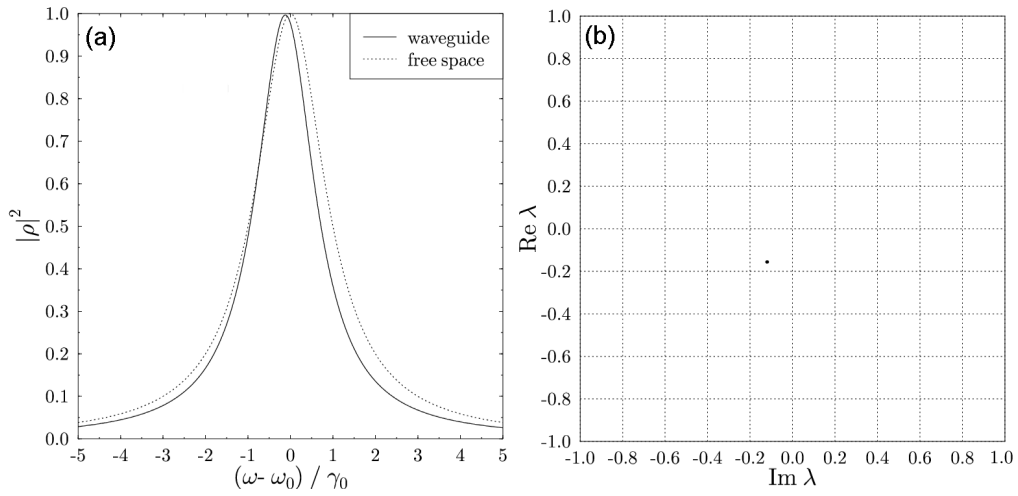

Fig. 1. (a) Reflection $R$ of a single dielectric cylinder placed in a planar metallic waveguide plotted as a function of the frequency $\omega$ and (b) the corresponding spectrum of the $G$ matrix. The dashed line corresponds to the transmission in a "free space" case.

According to Eqs. (23) the eigenvalues $\lambda$ can be considered as a first-order approximation (in $\gamma_{0} / \omega_{0}$ ) to the positions of the resonance poles in the system. Thus if $\lambda \neq 0$ then $\omega \neq \omega_{0}, \gamma \neq \gamma_{0}$. To check if the shape of the resonance has really changed in Fig. 1a we have plotted as a solid line the reflection coefficient $R=\left|\rho_{1}\right|^{2}$. The dashed line in the same plot corresponds to the "free space" case $\lambda=0$.

\section{Collective resonances}

In the next step in Figs. 2 and 3 we plot the reflection $R$ of the systems of $N=10,100$ cylinders as a function of the frequency $\omega$. In the same plots we have also the corresponding approximate values of the resonance poles given by the spectrum of the $G$ matrix. The cylinders were distributed randomly with constant uniform density $n=1$ cylinder per wavelength squared. Therefore for each $N$ the size of the system was proportional to the number of cylinders $L \propto N$.

We have seen that in the case $N=1$ the incident wave was totally reflected for a single value of $\omega=\tilde{\omega}$. Let us note that not necessarily $\tilde{\omega}=\omega_{0}$, and therefore for this value of $\omega$ the total scattering cross-section $\sigma$ of an individual dielectric cylinder (14) does not approach its maximal value. However, for systems containing $N=10$ and $N=100$ the entire regions of the values of frequencies $\omega$ exist for which $R \simeq 1$. They are separated by narrow maxima of transmission. Moreover, 

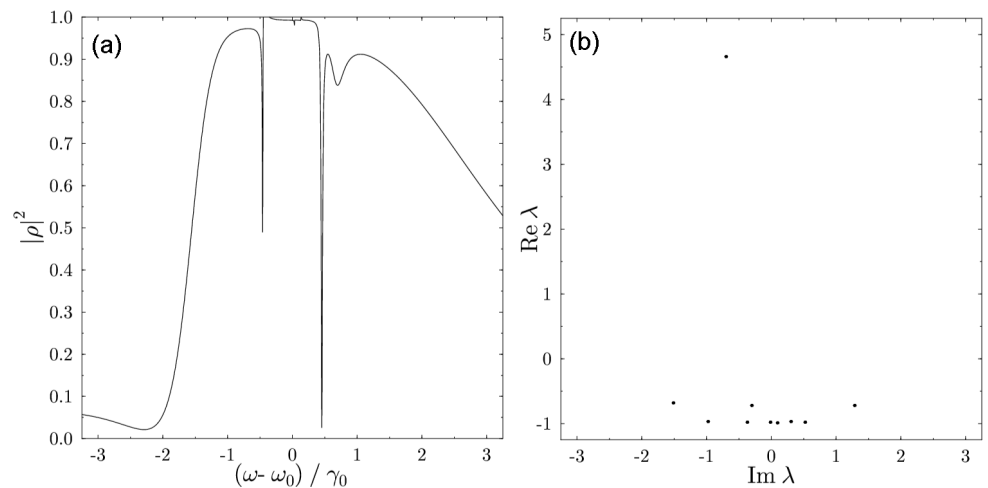

Fig. 2. (a) Reflection $R$ of a system of $N=10$ dielectric cylinders placed randomly in a planar metallic waveguide plotted as a function of the frequency $\omega$. (b) First-order approximations to the resonance poles in the system given by the spectrum $\lambda$ of the corresponding $G$ matrix.
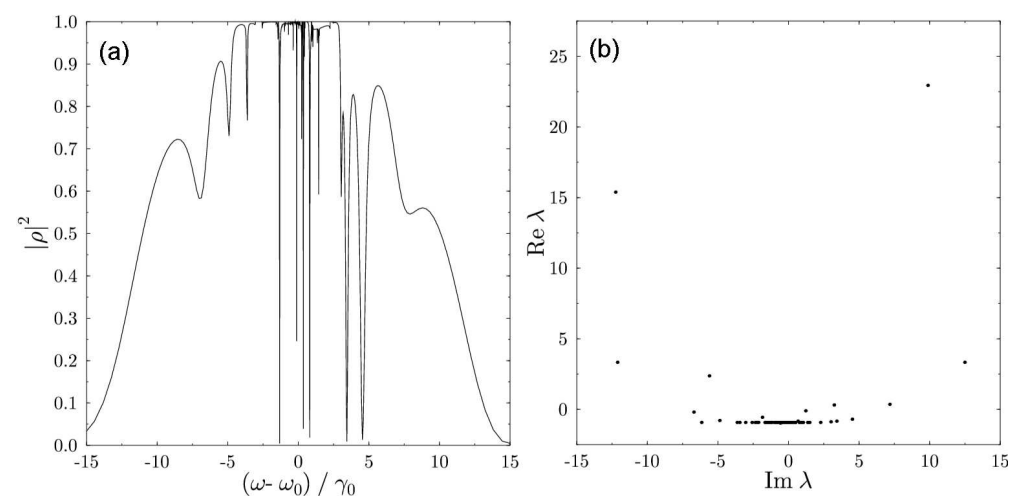

Fig. 3. (a) Reflection $R$ of a system of $N=100$ dielectric cylinders placed randomly in a planar metallic waveguide plotted as a function of the frequency $\omega$. (b) First-order approximations to the resonance poles in the system given by the spectrum $\lambda$ of the corresponding $G$ matrix.

inspection of Figs. 2 and 3 suggests that in the limit $N \rightarrow \infty$ the number of these maxima increases and simultaneously they became narrower and sharper. Therefore we may expect that for sufficiently large $N$ the incident waves will be totally reflected for almost any $\omega$ except the discrete set $\omega=\omega_{l}$ for which the reflection is close to unity. Physically speaking this means that different realizations of sufficiently large system of randomly placed cylinders are hardly distinguishable from each other by a transmission experiment.

It follows from inspection of Eqs. (9) and (10) that the maximum of transmission $T=\sum_{m}\left|\tau_{m}\right|^{2}=1$ (and minimum of reflection $R=\sum_{m}\left|\rho_{m}\right|^{2}=0$, because the medium is non-dissipative) corresponds to the case when the polarization of 
the medium fulfills the following condition:

$$
\sum_{a=1}^{N} p_{a} \mathcal{E}^{(0) *}\left(x_{a}, z_{a}\right)=\sum_{a=1}^{N} p_{a} \mathcal{E}^{(0)}\left(x_{a}, z_{a}\right)=0 \quad \text { for } \quad m=1, \ldots M .
$$

This means that in the expansion of the field radiated by the medium into waveguide modes the coefficients of near all guided modes Eq. (5) vanish. Therefore the radiated field consists only of evanescent modes with imaginary propagation constants $\beta_{m}$ and thus it is exponentially localized in the vicinity of the medium. In the next section we will show that such a field can exist also without any incident wave and therefore represents a truly localized wave.

\section{Anderson localization}

Let us note that in finite dielectric media no localized states are supported by Maxwell's equations in two dimensions [4]. However, this is not the case with confined media, where localized waves do exist even in finite media. Therefore a clear distinction between localized waves with isolated frequencies and the dense band of localized waves (due to the Anderson localization) is needed. We show now that this distinction may be provided by investigation of a phase transition which occurs in the limit of $N \rightarrow \infty$ in the spectra of $G_{a b}$ matrices corresponding to systems of randomly distributed dielectric cylinders.

To support this statement let us recall Figs. 2 and 3. We have plotted there the spectra $\lambda$ of a $G$ matrix (diagonalized numerically) corresponding to certain specific configurations of $N=10$ and $N=100$ cylinders placed randomly with the uniform density $n=1$ cylinder per wavelength squared. We see that already in the case of $N=10$ quite a lot of eigenvalues are located near the $\operatorname{Re} \lambda=-1$ axis. This tendency is more and more pronounced with increasing size of the system measured by $N$. This is a universal property of $2 \mathrm{D} G$ matrices, not restricted to this specific realization of the system only. To prove these statements we diagonalize numerically the $G$ matrix (16) for $10^{2}$ different distributions of $N=100$ cylinders. Then we construct two-dimensional histogram of eigenvalues $\lambda$ from all distributions. It approximates the corresponding probability distribution $P(\lambda)$ which is normalized in the standard way $\int \mathrm{d}^{2} \lambda P(\lambda)=1$. In Fig. 4 we have the surface plot of the function $P(\lambda)$. It clearly shows that for all configurations (without, may be, a set of zero measure) most eigenvalues are located near the $\operatorname{Re} \lambda=-1$ axis.

Our numerical investigations indicate that in the limit of an infinite medium, the probability distribution under consideration will tend to the delta function in the real part

$$
\lim _{N \rightarrow \infty} P(\lambda)=\delta(\operatorname{Re} \lambda+1) f(\operatorname{Im} \lambda) .
$$

This means that in this limit for almost any random distribution of the cylinders, an infinite number of eigenvalues satisfies the condition Eq. (21). It is therefore reasonable to expect that in the case of a random and infinite system a countable 


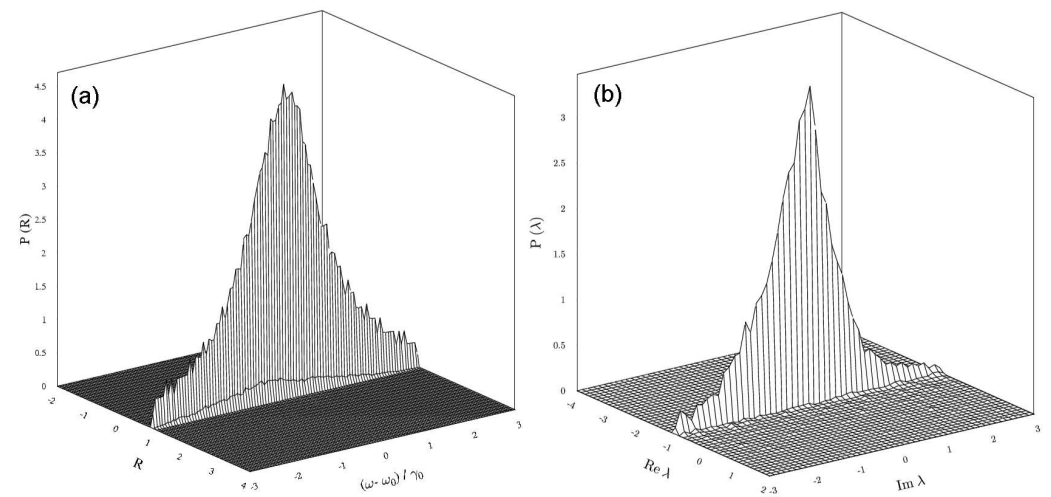

Fig. 4. (a) Probability $P(R)$ of measuring a reflection $R$ at frequency $\omega$. (b) Density of eigenvalues $P(\lambda)$ of the matrix $G$ calculated from $10^{2}$ distributions of $N=100$ cylinders calculated for the same systems. The similarity is striking.

set of frequencies $\omega_{l}$ corresponding to localized waves becomes dense in some finite interval. But it is always difficult to separate such frequencies from frequencies which may be arbitrarily near and physically the spectrum is always a coarse-grained object. Therefore in the limit of an infinite medium an entire band of spatially localized electromagnetic waves appears.

In addition in Fig. 4 we have the probability $P(R)$ of measuring a reflection $R$ at frequency $\omega$ calculated for the same systems of cylinders. This distribution is very similar to the distribution of eigenvalues. Thus incident waves are totally reflected for "almost any" frequency from the band of localized waves, i.e., except the discrete set (of zero measure) for which the transmission is equal to unity. This provides a connection between the phenomenon of localization and a dramatic inhibition of the propagation of electromagnetic waves in a spatially random dielectric medium.

\section{Summary}

In summary, we have developed a simple yet reasonably realistic theoretical approach to the Anderson localization of electromagnetic waves. A two-dimensional dielectric medium consisting of $N$ dielectric cylinders confined within a planar metallic waveguide has been studied. Several particular cases may be considered ( $k d=\pi$ is the cut-off thickness of the waveguide). For $N=0$ and $k d<\pi$ there are no guided modes in the waveguide as well as there are no localized waves. This case is analogous to the electronic band gap in a solid. If $N>0$ and $k d<\pi$, there are still no guided modes in the waveguide but localized waves can appear for any distribution of the cylinders. It is again analogous to the solid state physics situation where isolated perturbations of the periodicity of crystals (like impurities or lattice defects) can lead to the formation of localized electronic states with energies within the forbidden band. Another possibility corresponds to $N=0$ 
and $k d>\pi$. In this case there are guided modes but the system supports no localized waves. This is very similar to the conductance band in solids. Guided modes correspond to extended electronic states described by the Bloch functions. In this paper we perform a detailed study of the regime where $N>0$ and $k d>\pi$. For this range of parameters there are both the guided modes and the resonances of transmission. Isolated localized waves can be seen for certain distributions of the cylinders. The signs of the Anderson localization emerging in the limit of an infinite medium can be observed both in analysis of transmission and in the properties of the spectra of certain random matrices. Eventually we will consider a limiting case of $N \rightarrow \infty$ and $k d>\pi$. It turns out that in this case the guided modes no longer exist in the waveguide. Instead a band of localized waves will be formed for any distribution of the cylinders. It is an interesting analog of the Anderson localization in noncrystalline solids such as amorphous semiconductors or disordered metals.

\section{Acknowledgments}

This work was supported by the grant PBZ-KBN-044/P03/2001.

\section{References}

[1] D.S. Wiersma, P. Bartolini, A. Lagendijk, R. Righini, Nature 390, 671 (1997).

[2] A.Z. Genack, N. Garcia, Phys. Rev. Lett. 66, 2064 (1991).

[3] R. Dalichaouch, J.P. Armstrong, S. Schultz, P.M. Platzman, S.L. McCall, Nature 354, 53 (1991).

[4] M. Rusek, A. Orłowski, Phys. Rev. E 51, R2763 (1995).

[5] M. Rusek, A. Orłowski, J. Mostowski, Phys. Rev. E 56, 4892 (1997).

[6] J.D. Jackson, Classical Electrodynamics, Wiley, New York 1962.

[7] J.P. Dowling, M.O. Scully, F. DeMartini, Opt. Commun. 82, 415 (1991).

[8] J.P. Dowling, Found. Phys. 23, 895 (1993).

[9] B.A. Lippmann, J. Schwinger, Phys. Rev. 79, 469 (1950). 